\title{
Vertebral Body Erosion and Subsequent Back Pain Secondary to a Vena Cava Filter
}

\author{
William Newman $^{1}$, Nathan T. Zwagerman ${ }^{2}$, Peter C. Gerszten ${ }^{3}$ \\ 1. Neurological Surgery, Department of Neurological Surgery, University of Pittsburgh Medical Center 2. \\ Neurological Surgery, University Of Pittsburgh 3. Department of Neurological Surgery, University of \\ Pittsburgh Medical Center
}

$\square$ Corresponding author: William Newman, newmanwc@upmc.edu

Disclosures can be found in Additional Information at the end of the article

\section{Abstract}

Background: A patient with multiple open lumbar procedures, the most recent of which was complicated by symptomatic pulmonary embolus, underwent placement of an inferior vena cava filter (IVCF). Two years after placement, she developed low back pain with radicular symptoms. CT of the lumbar spine demonstrated vertebral body erosion from the IVCF strut. In this brief report, we describe L2 vertebral body erosion, radiographic findings, and a brief review of the literature.

Objective: To describe radiographic findings of vertebral body erosion from IVCF as well as review the literature regarding this and other complications of IVCF placement.

Methods: Retrospective, single patient chart review.

Results: The patient's IVCF was successfully removed without complication. The pain and radicular symptoms resolved by one month follow-up.

Conclusions: Based on the literature, most IVCFs with evidence of bony erosion are removed except when patient comorbidities, goals of care, or a complete absence of symptoms make removal inadvisable.

Received 12/25/2014 Review began 12/27/2014 Review ended 02/19/2015 Published 02/20/2015

\section{Copyright 2015}

Newman et al. This is an open access article distributed under the terms of the Creative Commons Attribution License CC-BY 3.0., which permits unrestricted use, distribution, and reproduction in any medium, provided the original author and source are credited.
Categories: Radiology, Neurosurgery

Keywords: inferior vena cava filter, vertebral body erosion

\section{Introduction}

A patient with multiple open lumbar procedures, the most recent of which was complicated by symptomatic pulmonary embolus, underwent placement of an inferior vena cava filter (IVCF). Two years after placement, she developed low back pain. CT of the lumbar spine demonstrated vertebral body erosion from the IVCF strut. In this brief report, we describe L2 vertebral body erosion, radiographic findings, and a brief review of the literature.

\section{Case Presentation}

The University of Pittsburgh Institutional Review Board issued approval IRB \# PRO08120394 for this case report. Informed patient consent was obtained for her treatment.

A patient had undergone several open lumbar procedures, including pedicle screw fixation. The 


\section{Cureus}

last surgery was complicated by a symptomatic pulmonary embolus. At that time, the patient underwent the uneventful placement of an inferior vena cava filter (IVCF). Two years after placement of the IVCF, the patient began having lower back pain with intermittent right flank pain. Four months later, the patient developed symptoms of urinary retention. The work-up for these symptoms included a CT of the lumbar spine. CT imaging demonstrated erosive changes of the L2 vertebral body with a prong of the IVCF violating the outer cortex (Figure 1A).
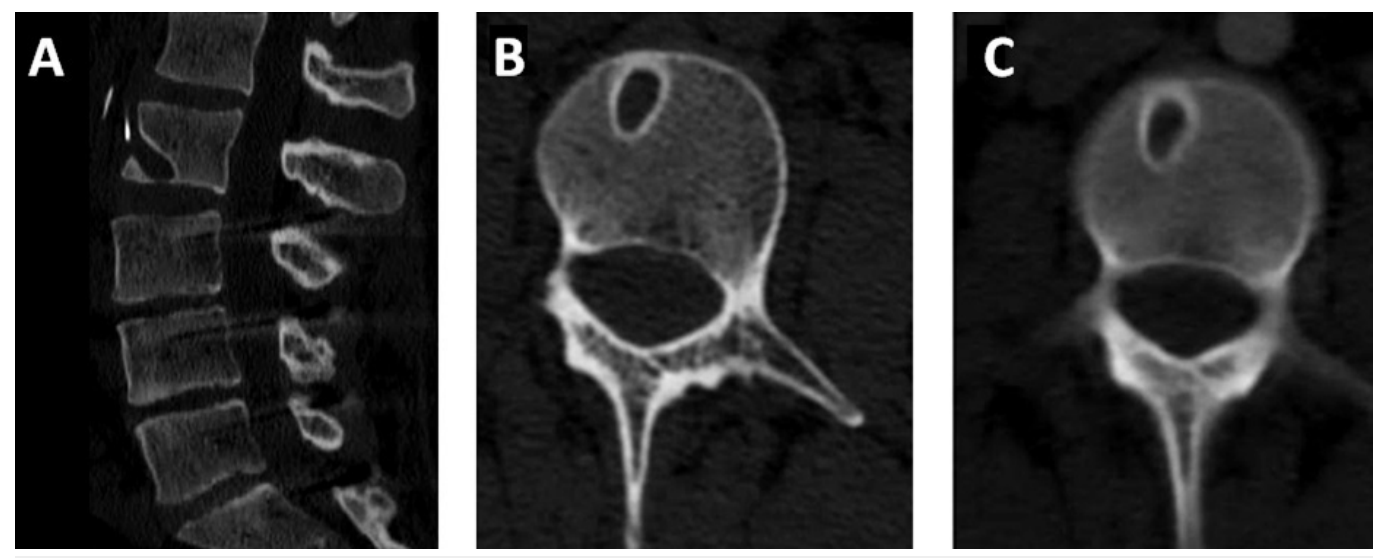

\section{FIGURE 1: Erosive changes of the L2 vertebral body}

CT imaging demonstrates erosive changes of the L2 vertebral body with a prong of the IVCF violating the outer cortex (Figure 1A).

A vascular surgery consultation recommended endovascular removal of the IVCF, which was performed without complication through an internal jugular approach. By one month followup, the new pain symptoms had resolved. Post-removal imaging demonstrated stable changes of the L2 vertebral body (Figure 1B, 1C).

\section{Discussion}

Inferior vena cava filter (IVCF) placement in patients at risk for thromboembolic events and contraindications to systemic anticoagulation is common. The literature demonstrates an overall complication rate of $10 \%$ [1], with rates of IVCF migration and fracture of less than $1.8 \%$ at six months and less than $4.3 \%$ at 12 months [2]. While complications involving the vertebral body are uncommon, these are not an unknown complication of IVCF placement [3-5]. However, vertebral body involvement alone is rarely seen. More frequently, such vertebral body involvement is associated with vascular complications, such as a pseudoaneurysm [6], retroperitoneal hematoma [7], or hematemesis from esophageal erosion [5]. The time to presentation amongst these case reports ranges from two days in the case of acute vascular complications [7] to 15 years for asymptomatic vertebral body erosion [4]. In our patient, the vertebral body erosion was found two years after IVCF placement due to the onset of symptoms of back pain and urinary retention, both of which improved after removal.

Despite evidence of bony erosion, these IVCF often are not removed secondarily to overall patient comorbidities, goals of care, or lack of symptoms [3-4, 8]. However, given the potential for adverse vascular and gastrointestinal complications as well as IVCF migration [2], removal of a no longer necessary IVCF should be attempted.

\section{Conclusions}


Based on the literature, most IVCFs with evidence of bony erosion are removed except when patient comorbidities, goals of care, or a complete absence of symptoms make removal inadvisable.

\section{Additional Information \\ Disclosures}

Human subjects: Consent was obtained by all participants in this study. University of Pittsburgh Institutional Review Board issued approval IRB \# PRO08120394. Conflicts of interest: In compliance with the ICMJE uniform disclosure form, all authors declare the following: Payment/services info: All authors have declared that no financial support was received from any organization for the submitted work. Financial relationships: All authors have declared that they have no financial relationships at present or within the previous three years with any organizations that might have an interest in the submitted work. Other relationships: All authors have declared that there are no other relationships or activities that could appear to have influenced the submitted work.

\section{References}

1. Wang W, Zhou D, Obuchowski N, et al.: Fracture and migration of celect inferior vena cava filters: a retrospective review of 741 consecutive implantations. J Vasc Interv Radiol. 2013, 24:1719-22. 10.1016/j.jvir.2013.07.019

2. Durack JC, Westphalen AC, Kekulawela S, et al.: Perforation of the IVC: Rule rather than exception after longer indwelling times for the Günther Tulip and Celect retrievable filters. Cardiovasc Intervent Radiol. 2012, 35:299-308. 10.1007/s00270-011-0151-9

3. Shaer JA, Epstein N: An unusual cause of low back pain? A case report . Spine (Phila Pa 1976). 1998, 15:1349-50.

4. Fang W, Hieb RA, Olson E, et al.: Asymptomatic lumbar vertebral erosion from inferior vena cava filter perforation. Cardiovasc Intervent Radiol. 2007, 30:494-6.

5. Salameh H, Sharif R, Larson SA, et al.: Duodenal perforation, vertebral body perforation, and aortic abutment after placement of retrievable inferior vena caval filter. Endoscopy. 2013, 45:E278-9. 10.1055/s-0033-1344323

6. Amole AO, Kathuria MK, Ozkan OS, et al.: Lumbar artery laceration with retroperitoneal hematoma after placement of a G-2 inferior vena cava filter. Cardiovasc Intervent Radiol. 2008, 31:1257-9. 10.1007/s00270-008-9365-x

7. Skeik N, McEachen JC, Stockland AH, et al.: Lumbar artery pseudoaneurysm caused by a Gunther Tulip inferior vena cava filter. Vasc Endovascular Surg. 2011, 45:756-60.

10.1177/1538574411419373

8. Cuadra SA, Sales CM, Lipson AC, et al.: Misplacement of a vena cava filter into the spinal canal. J Vasc Surg. 2009, 50:1170-2. 10.1016/j.jvs.2009.06.024 